\section{Zero contrast angioplasty of LAD ostium by marker wire technique}

\begin{abstract}
:
Zero contrast Angioplasty brings new hope and new era in treating patient with chronic kidney disease (CKD). It is technically very promising approach for the prevention of contrast induced nephropathy (CIN). Contrast induced nephropathy (CIN) is a common iatrogenic complication associated with adverse outcome including need for Renal replacement therapy (RRT). Zero contrast $\mathrm{PCl}$ is creating new era in the field of coronary intervention specially treating those patients with CKD who are suffering from refractory angina and not indicated or fit for Cardiac Bypass surgery. Besides treating simple lesion, challenging case like LAD ostial $\mathrm{PCl}$ with placement of stent precisely in LAD ostium can be done by zero contrast or minimal contrast. In this case, for the first time marker wire technique had been used with applying some innovative approach in placing stent precisely in ostium successfully.
\end{abstract}

Keywords: Zero contrast angioplasty $\square$ Marker wire technique $\backsim$ LAD ostial angioplasty

\section{Abbreviation list:}

IVUS: Intravascular Ultrasound; FFR/IFR: Fractional flow reserve/Instantaneous wave free ratio; MI: Myocardial infarction; CAG: Coronary Angiogram; LCX: Left Circumflex; RAO: Right Anterior Oblique; LAO: Left Anterior Oblique; EBU: Extra Back up; LM: Left Main; AKI: Acute Kidney Injury

\section{Introduction}

Contrast-induced nephropathy is a serious complication after intravascular administration of iodinated contrast media and is associated with numerous adverse outcomes. Its prevalence is particularly high in patients with multiple comorbidities who undergo coronary angiography and percutaneous coronary intervention (PCI) [1]. Zero contrast Angioplasty is a technically promising approach for the prevention of contrast induced nephropathy. Contrast induced nephropathy (CIN) potentially preventable. Despite of some established approaches to prevent CIN include peri-procedural hydration [2] and minimizing contrast volume [3] percutaneous coronary intervention $(\mathrm{PCl})$ in patients with advanced $\mathrm{CKD}$ is associated with a high risk of $\mathrm{CIN}$ and requirement for RRT, leading to underutilization of $\mathrm{PCl}$ in these high-risk patients. With the development of expertise in advance technology like IVUS, FFR/IFR it is possible to do even complex PCI by 'minimal' or 'zero' contrast successfully with successful stent implantation. Here we did LAD ostial PCl with precise placement of stent in ostium with using no contrast. Very simple but innovative technique "Marker Wire Technique" were used for precise placement of stent to ostium.
Afzalur Rahman', Farhana Ahmed $^{2}$, Mohammad Arifur Rahman $^{3^{*}}$

'Professor and Ex. Director, National Institute of cardiovascular Diseases, Sher e Bangla Nagar, Dhaka, Bangladesh

${ }^{2}$ Assistant Professor, Cardiology, National Institute of cardiovascular Diseases, Sher e Bangla Nagar, Dhaka, Bangladesh 3Junior Consultant, Cardiology, National Institute of cardiovascular Diseases, Shere Bangla Nagar, Dhaka, Bangladesh

*Author for correspondence: E-mail: Drarif79@yahoo.com Received date: April 05, 2020 Accepted date: April 15, 2020 Published date: April 22, 2020 


\section{Clinical background:}

A 50 years old young man with a history of hypertension, and CKD Stage IV with an estimated glomerular filtration rate (eGFR) of $20 \mathrm{ml} / \mathrm{min} / 1.73 \mathrm{~m} 2$ (normal: $\geq 60 \mathrm{ml} / \mathrm{min} / 1.73 \mathrm{m2}$ ) presented with recent $\mathrm{MI}$ anterior with ongoing chest pain and identified wall motion abnormality in antero-septal wall. Ultra contrast Angiography was done and demonstrated significant lesion in mid LAD (Figure 1).

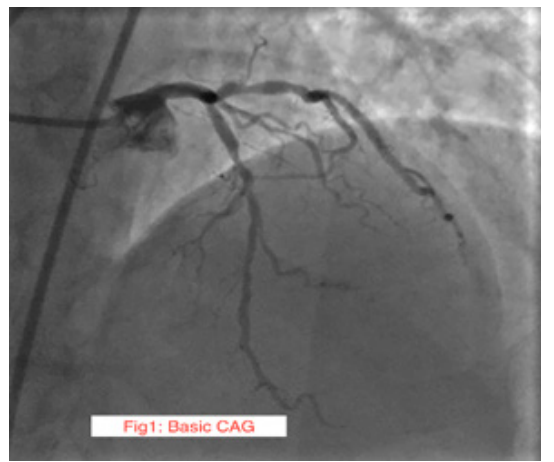

Figure 1: Basic Angiogram

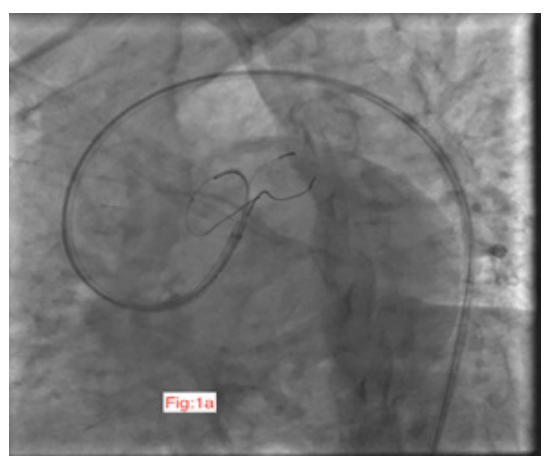

Figure 1a: Placement of wire in LAD and LCX

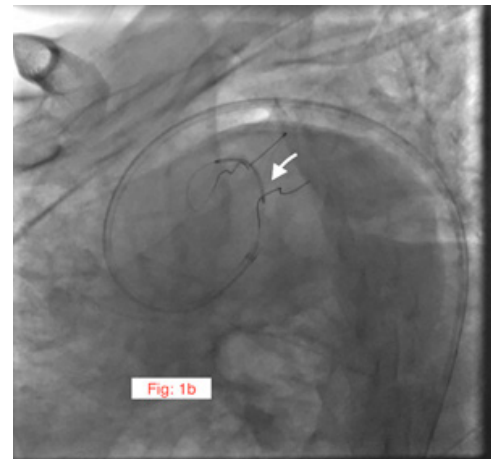

Figure 1b: Placement of $L A D$ stent in the ostium

\section{Procedural description:}

Lesion was looking simple apparently and we planned to do $P C l$ to LAD 10 days after diagnostic CAG. Prior to the procedure, previous angiographic films were uploaded to the monitors as a guide for coronary wiring. We prefer RAO cranial and LAO caudal view of Left coronary artery as reference. Using those images as reference EBU guide catheter was engaged to LM ostium. Three floppy wire, one was negotiated through the lesion to distal LAD and another two wires, one was in diagonal 1, another wire was in LCX (Figure 1a).

Diagonal wire and LCX wire were also play role as landmark wire, IVUS was done. IVUS run found significant plaque in LAD ostium too and LM is free of disease. So apparently, a simple case turned into a complex one. We decided to place stent precisely in the LAD ostium as LM is disease free. During IVUS run, we select distal landing zone and approximate length of stent. DES $2.5 \times 38 \mathrm{~mm}$ was deployed covering the lesion of mid LAD. At this, point LCX wire was pulled back and marker portion of the wire was kept in LM to LCX to make a metallic silhouette (Figure 1b). The idea was that with blood flow the softer portion of wire will stuck with the roof of carina, which acts as marker of ostium. DES $3 \times 20 \mathrm{~mm}$ (Figure $1 \mathrm{~b}$ ) was placed to the LAD ostium and deployed. After deployment of stent and post dilatation IVUS was done. IVUS also confirm the placement of stent precisely LAD ostium by this technique. Final check (Figure 2) was taken by using $2 \mathrm{ml}$ of dye to keep

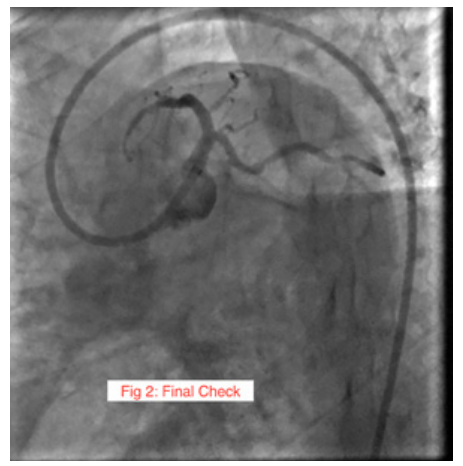

Figure 2: Final view after stenting

record and check for any complication. Total $2 \mathrm{ml}$ dye was used to finish entire procedure.

\section{Discussion:}

Among high-risk patient subset, patients with advanced CKD are at an even greater risk of adverse events, as CIN is directly correlated with higher rates of adverse clinical events and progression to end stage renal disease [4]. Performing minimal or zero contrast $\mathrm{PCl}$, when indicated, offers the opportunity to improve overall outcomes related to ischemic heart disease without risking worsening renal function or dialysis in this extreme risk group of patients [5]. Careful 
study of the prior diagnostic angiogram is fundamental to successful wire navigation without using contrast. Significant expertise with IVUS and coronary physiological technology is also quintessential and only highly trained IVUS interpreters operators should attempt these procedures [6].

Our strategy of performing zero contrast $\mathrm{PCl}$, staged after ultra contrast diagnostic angiography, facilitates procedural planning as well as staged $\mathrm{PCl}$ also allows time for better discussion with patients regarding the treatment options, allowing true informed consent to be obtained. We chose to perform the staged $\mathrm{PCl}$ no earlier than 7 days after the angiography because CIN typically occurs within the first week after the contrast exposure [2]. Careful study of the prior diagnostic angiogram is fundamental to successful wire navigation without using contrast. Significant expertise with IVUS and coronary physiological technology is also quite essential and only highly trained IVUS interpreter operators should attempt these procedures. Besides that, single injection of small amount (2-3 ml) of contrast into coronary artery in order to exclude complications (especially distal perforation or embolization) and document final angiographic result.

In this case one of its biggest challenge is the precise positioning of stents in LAD ostium. In our case, During IVUS manual pull back, after determining the safe landing zone we kept simultaneously scene record of position of IVUS transducer that correspond to the safe landing zone (Figure: $3 \mathrm{a}, 3 \mathrm{~b})$. Besides that, multiple wiring of side branch acts as a landmark. Keeping the softer marker part of wire in LM to LCX make a metallic silhouette. The idea was that with blood flow the softer portion of wire will stuck with the roof of carina, which acts as marker of LAD ostium. As LM is free of disease, we prefer here precise placement of stent in LAD ostium to prevent undue jailing of LCX as well as metal in LM. The case demonstrates that by marker wire technique precise placement of stent to LAD ostium is possible.
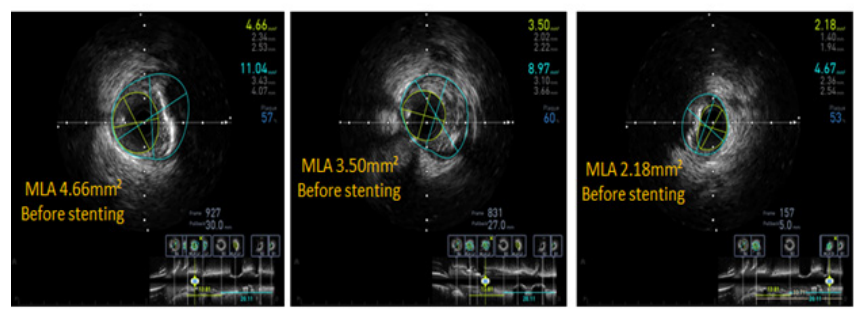

LAD Proximal

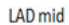

LAD distal
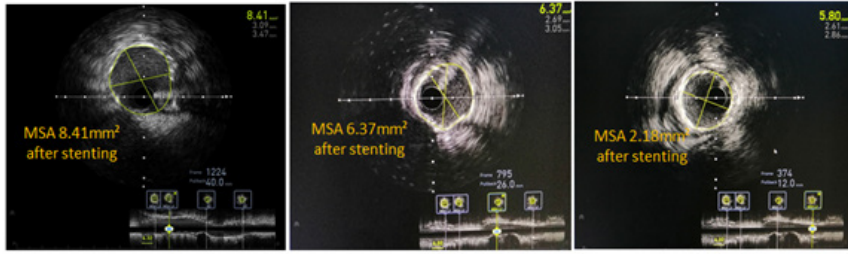

LAD Proximal LAD mid

LAD distal

Figure 3b: IVUS study after stenting

For such procedure, a high level of preparedness and expertise in managing coronary complications is necessary. Close monitoring of these patients during the peri-procedural period is essential.

\section{Conclusion}

In conclusion, zero contrast $\mathrm{PCl}$ that can be utilized in experienced centers with expert operator safely to revascularize selected high-risk patients with advanced CKD or history of Contrast induced AKI. Complex case like LAD ostial lesion can also treated successfully by using marker wire technique.

\section{Conficts of Interest:}

All authors have no financial/proprietary interest in the subject matters of manuscript.

\section{References}

1. Sacha J, Gierlotka M, Feusette P, et al. Ultra-low contrast coronary angiography and zero-contrast percutaneous coronary intervention for prevention of contrast-induced nephropathy: step-by-step approach and review. Postepy Kardiol Interwencyjnej. 15(2): 127-136 (2019).

2. Brar SS, Aharonian V, Mansukhani P, et al. Haemodynamic-guided fluid administration for the prevention of contrast-induced acute kidney injury: the POSEIDON randomised controlled trial. Lancet. 383(9931): 1814-1823 (2014).

3. Mehran R, Nikolsky E. Contrast-induced nephropathy: definition, epidemiology, and patients at risk. Kidney Int Suppl. 69(100): S11-S15 (2006).

4. Giacoppo D, Madhavan MV, Baber U, et al. Impact of contrast-induced acute kidney injury after percutaneous coronary intervention on shortand long-term outcomes: pooled analysis from the HORIZONS-AMI and ACUITY Trials. Circ Cardiovasc Interv. 8(8): e002475 (2015)

5. Ali ZA, Karimi Galougahi K, Nazif T, et al. Imaging and physiology-guided percutaneous coronary intervention without contrast administration in advanced renal failure: a feasibility, safety, and outcome study. Eur Heart J. 37(40): 3090-3095 (2016).

6. Hatem R, Finn MT, Riley RF, et al. Zero contrast retrograde chronic total occlusions percutaneous coronary intervention: a case series. Eur Heart J Case Rep. 2(2): yty036 (2018).

Figure 3a: IVUS study before stenting 